\title{
Workspace and Stiffness Analysis of 3D Printing Cable-Driven Parallel Robot with a Retractable Beam-Type End-Effector
}

\author{
Jinwoo Jung \\ School of Electronic and Electrical Engineering, Daegu Catholic University, \\ Gyeongsan-si 38430, Gyeongsangbuk-do, Korea; jwjung11@cu.ac.kr
}

Received: 14 July 2020; Accepted: 21 August 2020; Published: 24 August 2020

\begin{abstract}
D printing is a widely used technology that has been recently applied in construction to reduce construction time significantly. A large 3D printer often uses a traditional Cartesian robot with inherent problems, such as position errors and printing nozzle vibrations, due to the long, heavy horizontal beam carrying it and a large amount of power required to actuate the heavy beam. A cable-driven parallel robot (CDPR) can be a good alternative system to reduce the vibrations and necessary power because the robot's lightweight cables can manipulate the printing nozzle. However, a large 3D printing CDPR should be carefully designed to maximize the workspace and avoid cable interference. It also needs to be stiff enough to reject disturbances from the environment properly. A CDPR with a retractable beam-type end-effector with cables through the guide pulleys in a single plane is suggested for avoiding cable interference while maximizing the workspace. The effects of using the retractable end-effector on the workspace were analyzed relative to the cable connection points' location changes. Static stiffness analysis was conducted to examine the natural frequencies, and the geometric parameters of the end-effector were adjusted to improve the lowest natural frequencies. Simulation results show that a retractable beam-type end-effector can effectively expand the wrench-feasible workspace.
\end{abstract}

Keywords: cable-driven parallel robot; retractable end-effector; workspace; stiffness; cable interference

\section{Introduction}

The application of 3D printing technology has been improved and expanded, including in the production of traditional plastics and metallic parts [1,2] to bio-printing objects [3]. Recently, the application of 3D printing technology has expanded further into large construction sites [4,5], as it can significantly reduce material consumption because materials may only be used where they are needed while reducing construction time by printing out complex structures without time-consuming assembly processes. One popular system for implementing a 3D printer is a Cartesian robot that consists of horizontal and vertical beams. However, using a Cartesian robot introduces several problems. First, the horizontal beam carrying the printing nozzle tends to bend because of the nozzle's weight. Second, when the 3D printer's size increases, the horizontal beam becomes long and heavy, which induces considerable bending and position errors when printing large structures.

Another promising system for 3D construction printing is a cable-driven parallel robot (CDPR) because it uses lightweight cables to manipulate an end-effector (Figure 1). A CDPR covers a large area because the robot provides a scalable workspace by changing the locations of its guide pulleys. There have been several studies on the use of CDPRs for large 3D construction printing [6-8]. However, developing a CDPR for 3D construction printing requires a careful design process because its design parameters can significantly affect workspace volume, cable interference with a printed structure, 
and robot stiffness. Most of the researchers for 3D construction printing have mainly focused on the development of a 3D-printing CDPR for construction, followed by the research of the investigation of the 3D-printing accuracy and the improvement based on the kinematic calibration. For most of the previously developed 3D-printing CDPRs for construction, suspended cable connection has been typically selected for securing the workspace under the end-effector since there can be no cables passing under the end-effector. A large suspended CDPR for construction, called Control of Giant Robots (CoGiRo), was developed and tested for the printing accuracy of a large wall [6]. A 3D printing CDPR was developed and the feasibility of its mechanism was investigated using simulation and experiments [7]. The kinematic calibration method for a 3D-printing CDPR had been researched for improving the accuracy of the robot [8]. The kinematics and statics of the 12-cable-driven robot with the eight lower cables had been investigated [9]. A suspended CDPR combining CoGiRo [6] and the 12-cable-driven robot [9] had been developed and its performance has been evaluated in terms of enhancing the quality of the largely printed object [10]. However, a suspended CDPR has a significant disadvantage that it does not have vertical stiffness since the end-effector is suspended vertically by the gravitational force and not explicitly constrained by cables. Thus, if a suspended CDPR is exposed to vertical disturbances from the environment, such as vibration from a rock drill or sudden gust of wind, its accuracy can be considerably degraded.

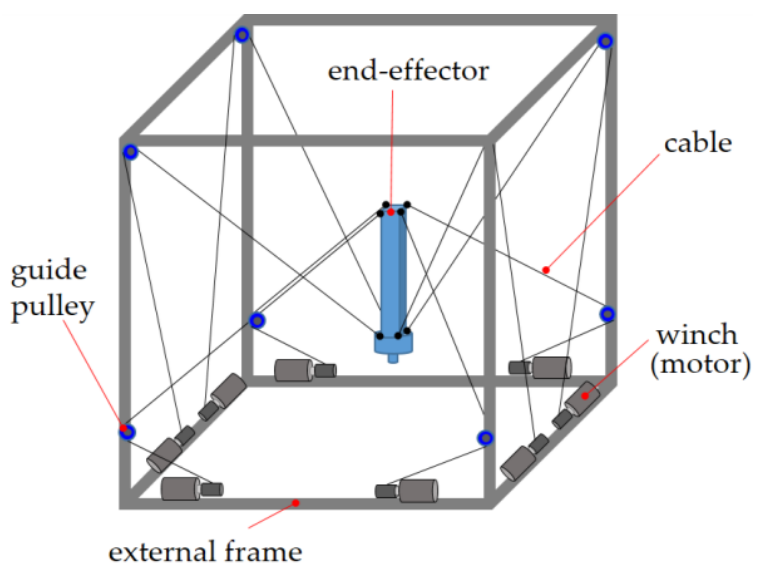

Figure 1. An eight-cable vertically crossed over-constrained cable-driven parallel robot (CDPR).

In order to have vertical stiffness, the cables that provide the end-effector with vertical forces can be additionally connected to the end-effector. In general, there are two methods of connecting cables for providing the vertical forces: one is to connect the cables to the bottom tip of the end-effector and guide them through the guide pulleys at the bottom of the external frame and the other is to connect the cables to the top of the end-effector and guide them through the guide pulleys at the bottom of the external frame. The latter method is a vertically crossed cable connection as shown in Figure 1. While the use of the former method can easily cause the cable interference with a printed object due to the cables fixed to the bottom tip of the end-effector, the latter method can allow more workspace than the former method. However, the latter method still has the cables going toward the bottom of the external frame and eventually leads to the cable interference with a printed object. For example, a 3D-printing CDPR using the vertically crossed cables can have the cable interference problem as shown in Figure 2. Considering the 3D-printing CDPR printing a tall and wide object, the locations of the guide pulleys near the bottom may become lower than the height of the object being printed. Along with the height increase of the printed object, when the 3D-printing CDPR tries to print a corner of the object, the cables coming from the guide pulleys near the bottom will interfere with the opposite corner of the printed object as shown in Figure 2. 


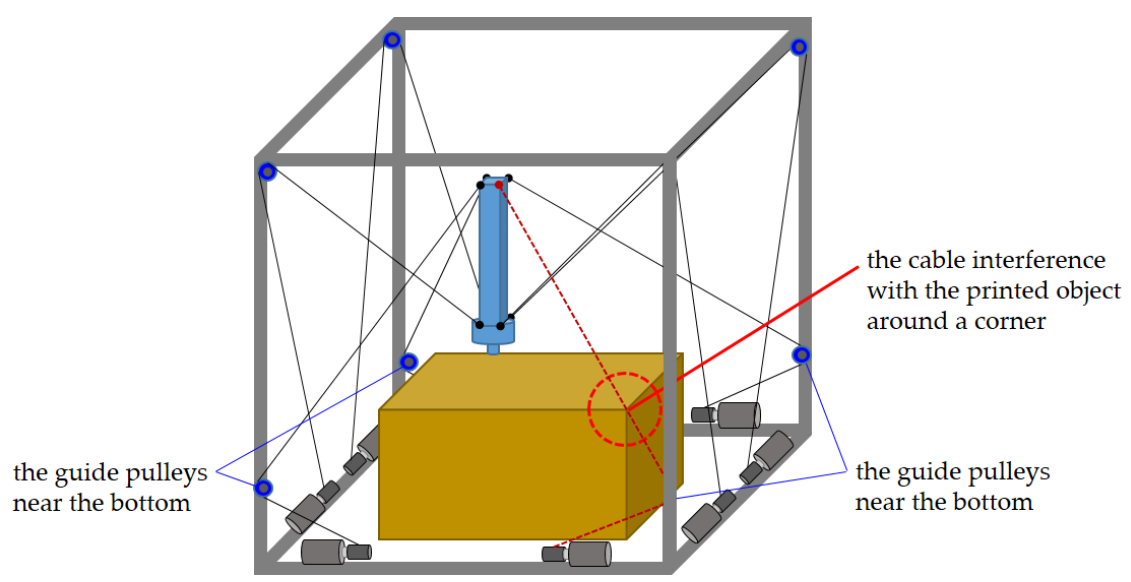

Figure 2. The cable interference with the printed object around a corner.

In order to solve the cable interference problem with the vertically crossed cables, the guide pulleys near the bottom can be lifted up to the top plane as shown in Figure 3a. The height increase of the guide pulleys can prevent the cables from occupying the workspace under the end-effector while maintaining vertical stiffness. Along with the guide pulleys at the top plane, using the short end-effector at the high positions can be advantageous to expand the horizontal workspace. This is because the horizontal component of cable force increases with the use of the short end-effector as shown in Figure 3b. In addition, the long end-effector is useful to reach low positions as shown in Figure 3c.

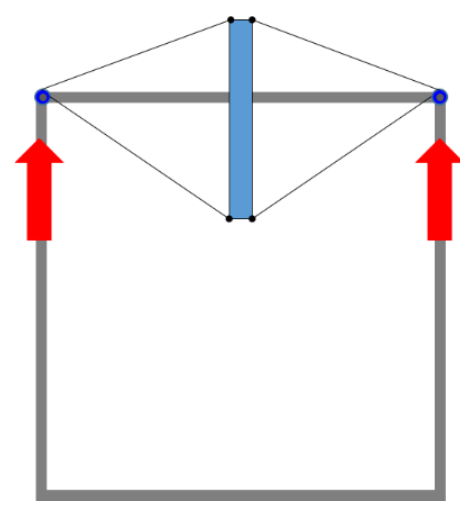

(a) The end-effector with the guide pulley in the top plane

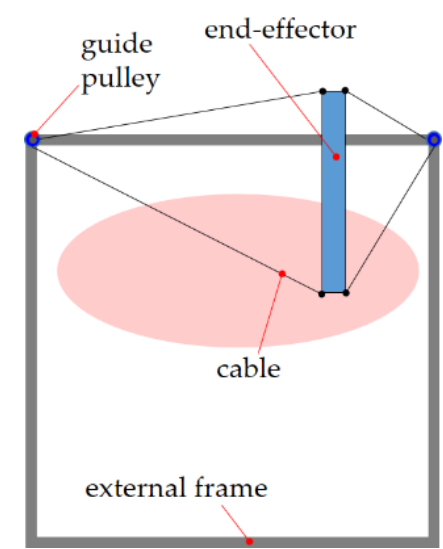

(b) The short end-effector for the high positions

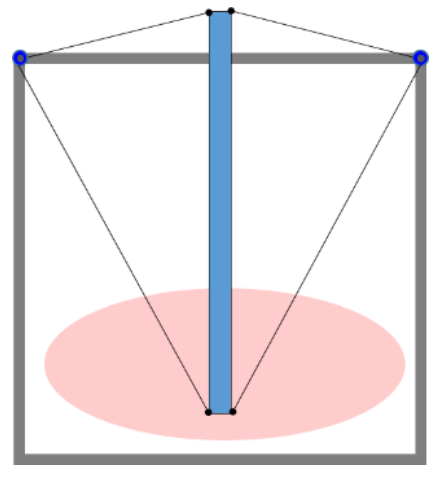

(c) The long end-effector for the low positions

Figure 3. The different lengths of the end-effector with the guide pulleys in the top plane.

Therefore, in this paper, for addressing the cable interference problem with maximizing the workspace volume while providing vertical stiffness, a novel retractable beam type end-effector with the guide pulleys distributed in a single horizontal plane is proposed for a 3D-printing CDPR as shown in Figure 4. The cable interference with the objects printed in workspace can be avoided by the cables guided thorough the guide pulleys in the horizontal top plane in Figure 4. This is because there is no cable cutting across the workspace from the guide pulley at the bottom of the external frame to the end-effector as compared to vertically crossed cable connection in Figure 1. To the best of the author's knowledge, there has been no attempt to use a retractable end-effector with the guide pulleys in the same plane for 3D-printing application. 


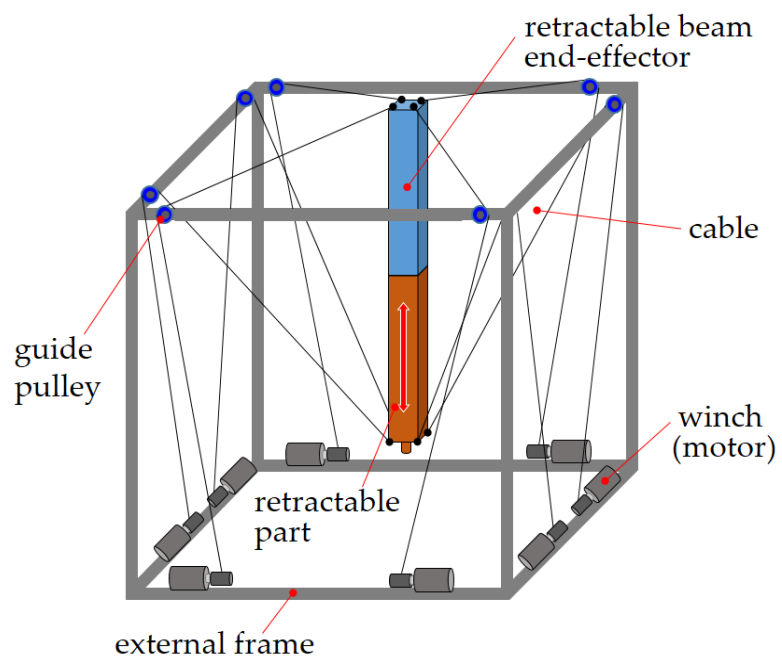

Figure 4. A cable-driven parallel robot with a retractable beam-type end-effector.

The use of the proposed end-effector should be investigated by conducting the workspace analysis and stiffness analysis for CDPRs as the workspace analysis of CDPRs has been conducted considering feasible tension distributions for various CDPRs [11-14]. In addition, the stiffness analysis of CDPRs has been researched for several CDPRs [15-18].

One of the main goals of this paper is to figure out how the length change of the retractable end-effector can be optimized with respect to maximizing the workspace for 3D-printing. Another important goal is to investigate the effectiveness of a design method of adjusting the geometry of the end-effector to increase the lowest natural frequencies in order to make the 3D-printing CDPR robust to the external disturbances. Through conducting a series of simulations, it is uniquely depicted that the wrench feasible workspace can be expanded by changing the length of the retractable end-effector with the guide pulleys in a single plane with respect to the z-position changes of the end-effector. In addition, the improvement of the lowest natural frequencies of the CDPR is newly achieved with respect to the length change of the end-effector along with changing the z-position by the geometry change of end-effector.

This paper is structured as follows: For evaluating the effectiveness of a CDPR with a retractable beam type end-effector having the guide pulleys in a single plane, workspace and static stiffness analysis have been conducted. In the first section, inverse kinematics and equations of motion for a CDPR are briefly introduced. Secondly for workspace analysis, the effects of the distribution of the guide pulleys (cable connection points on the external frame) on the size of workspace is analyzed and the effectiveness of changing the length of the retractable end-effector on the workspace has been investigated. Then, static stiffness analysis is conducted to investigate the natural frequencies for the spatial translation and rotation motions (six degrees of freedom (DOFs) of a CDPR) and the geometric parameters of the end-effector are modified for improving the lowest natural frequencies. Simulations successfully show that the CDPR using a proposed retractable beam mechanism can be effective for 3D construction printing.

\section{Inverse Kinematics and Equations of Motion for a CDPR with a Retractable End-Effector}

The eight-cable setup for the CDPR with a retractable end-effector was chosen to ensure a symmetrical 3D printing workspace. Inverse kinematics of the retractable CDPR was calculated by using the parameters based on the origin coordinate $F_{0}$ in Figure 5 , where $\mathbf{l}_{i}$ is the cable length vector to the $i$-th cable connection point (the location of the guide pulley) on the external frame. Meanwhile, $\mathbf{p}$ is the position vector to the end-effector's center of mass, which indicates the position of the printing nozzle. $\mathbf{R}_{e}$ is the rotation matrix representing the rotation of the end-effector relative to $F_{0}$, while $\mathbf{a}_{i}$ is the position vector from $F_{o}$ to the $i$-th cable connection point on the external frame. Lastly, $\mathbf{b}_{i}$ is the 
vector from the end-effector's center of mass to the cable connection point on the end-effector. Thus, the cable length vectors corresponding to the position of the end-effector can be calculated by using the vector loop Equation (1).

$$
\mathbf{l}_{i}=\mathbf{a}_{i}-\mathbf{R}_{e} \mathbf{b}_{i}-\mathbf{p}, \quad i=1, \ldots, 8
$$

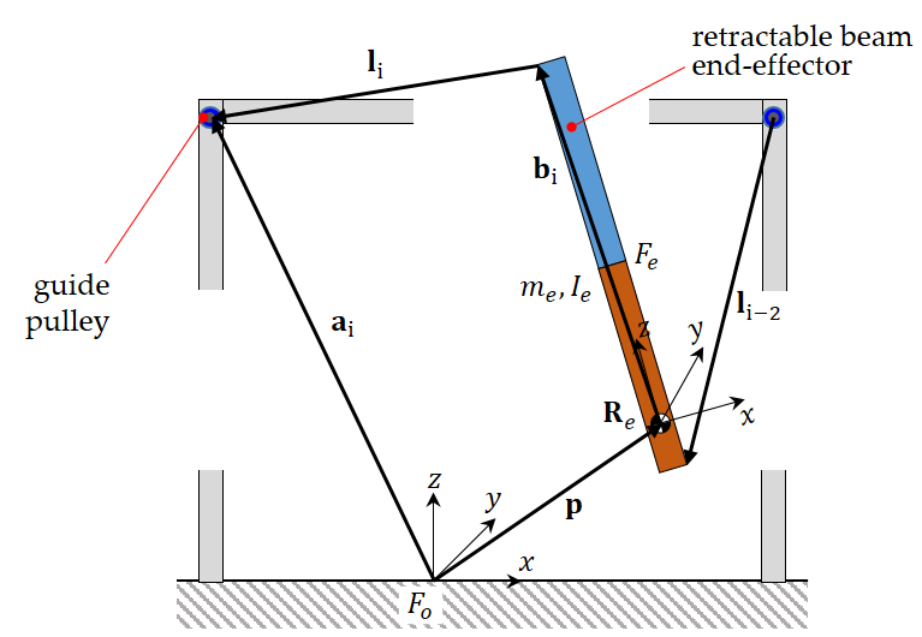

Figure 5. Geometric parameters of a cable-driven parallel robot (CDPR) with a retractable beam-type end-effector.

Cable length vector $\mathbf{1}_{i}$ is normalized by length $\left|\mathbf{1}_{i}\right|$, and it is used to derive a structure matrix corresponding to the end-effector's position (Equation (2)).

$$
\mathbf{u}_{i}=\mathbf{1}_{i} /\left|\mathbf{1}_{i}\right|, \quad i=1, \ldots, 8
$$

Equation (3) shows the relationship between the wrench, $\mathbf{w}_{\boldsymbol{e}}$, and cable force vector, $\mathbf{f}_{c}$, including the structure matrix, $\mathbf{A}^{\mathrm{T}}$. The wrench, $\mathbf{w}_{\boldsymbol{e}}$, includes translational force vector, $\mathbf{f}_{e}$, and moments, $\mathbf{t}_{e}$, to the end-effector.

$$
\mathbf{w}_{e}=\left[\begin{array}{c}
\mathbf{f}_{e} \\
\mathbf{t}_{e}
\end{array}\right]=-\mathbf{A}^{\mathrm{T}} \mathbf{f}_{\mathcal{C}}=-\left[\begin{array}{ccc}
\mathbf{u}_{1} & \ldots & \mathbf{u}_{\mathrm{n}} \\
\mathbf{b}_{1} \times \mathbf{u}_{1} & \ldots & \mathbf{b}_{\mathrm{n}} \times \mathbf{u}_{\mathrm{n}}
\end{array}\right] \mathbf{f}_{\mathcal{C}}, \quad \mathrm{n}=1, \ldots, 8
$$

Equations of motion for a CDPR in a matrix form with mass and stiffness matrices can be used for stiffness analysis to calculate the natural frequencies of a CDPR. In Equation (4), equations of motion are written in a matrix form using mass and stiffness matrices that include the end-effector's mass and inertia tensors and the cables' stiffness, respectively. The stiffness matrix in Equation (4) is represented as a cable stiffness matrix (Equation (7)) multiplied by the structure matrix, $\mathbf{A}^{\mathrm{T}}$, and the transposition of the structure matrix. The matrix $\mathbf{K}$ is a diagonal matrix whose elements are the $i$-th cables' stiffness (Equation (7)). The cable force distribution, $\mathbf{f}_{\mathcal{C}}$, is calculated using the closed-form method and written as Equation (8) [19]. In Equation (9), stiffness matrix multiplication can be derived by using wrench change relative to the cable force change (Equation (10)), cable force change relative to cable elongation change, $\Delta \mathbf{q}$, (Equation (11)), and the relationship between cable elongation rate and end-effector velocity (Equation (12)). Equation (4) is simplified by the assumption that most 3D printing motions are relatively slow, and the location of the end-effector's center mass is close to the bottom and maintained in a way in which the centrifugal and Coriolis forces are negligible during retraction. The center of mass is assumed to be near the bottom because there are several 3D printer components located in the area, including the printing nozzle, cooling fan, and an extruding motor.

$$
\mathbf{M}_{e} \ddot{\mathbf{x}}_{p}+\mathbf{A}^{\mathbf{T}} \mathbf{K} \mathbf{A} \mathbf{x}_{p}=-\mathbf{A}^{\mathbf{T}} \mathbf{f}_{\mathcal{C}}
$$




$$
\begin{gathered}
\mathbf{M}_{e}=\left[\begin{array}{cc}
\mathbf{m}_{e} & 0 \\
0 & I_{e}
\end{array}\right], \quad \mathbf{m}_{e}=\left[\begin{array}{ccc}
m_{e} & 0 & 0 \\
0 & m_{e} & 0 \\
0 & 0 & m_{e}
\end{array}\right], \quad I_{e}=\left[\begin{array}{ccc}
I_{x} & 0 & 0 \\
0 & I_{y} & 0 \\
0 & 0 & I_{z}
\end{array}\right], \\
I_{x}=\frac{1}{12} m_{e}\left(\left(y_{e}\right)^{2}+\left(z_{e}\right)^{2}\right), \quad I_{y}=\frac{1}{12} m_{e}\left(\left(x_{e}\right)^{2}+\left(z_{e}\right)^{2}\right), \quad I_{z}=\frac{1}{12} m_{e}\left(\left(x_{e}\right)^{2}+\left(z_{e}\right)^{2}\right), \\
\mathbf{K}=\operatorname{diag}\left(k_{1}, \ldots, k_{n}\right), \mathbf{n}=1, \ldots, 8 \\
\mathbf{f}_{c}=\mathbf{f}_{M}-\mathbf{A}^{+\mathrm{T}}\left(\mathbf{w}_{e}+\mathbf{A}^{\mathrm{T}} f_{M}\right), \mathbf{f}_{M}=\left(f_{\text {min }}+f_{\text {max }}\right) / 2
\end{gathered}
$$

The mass of the end-effector is $m_{e}$ and inertia, $I_{x}, I_{y}, I_{z}$ along each axis are calculated by using the dimensions of the end-effector, $x_{\mathrm{e}}, y_{\mathrm{e}}, z_{\mathrm{e}}$. Meanwhile, $x_{e}, y_{e}$, and $z_{e}$ are the end-effector's width, depth, and length, respectively.

$$
\begin{aligned}
\Delta \mathbf{w}_{e}=-\mathbf{A}^{\mathrm{T}} \Delta \mathbf{f}_{c}=-\mathbf{A}^{\mathrm{T}} \mathbf{K} \Delta \mathbf{q}=-\mathbf{A}^{\mathrm{T}} \mathbf{K} \mathbf{A} \Delta \mathbf{x}_{p} \\
\Delta \mathbf{w}_{e}=-\mathbf{A}^{\mathrm{T}} \Delta \mathbf{f}_{c} \\
\Delta \mathbf{f}_{c}=\mathbf{K} \Delta \mathbf{q} \\
\Delta \mathbf{q} / \Delta t=\mathbf{A} \Delta \mathbf{x}_{p} / \Delta t
\end{aligned}
$$

\section{Workspace Analysis}

For workspace analysis, the calculation for the wrench-feasible workspace was conducted within a $2 \times 2 \times 2 \mathrm{~m}^{3}$ external frame. It is determined by calculating the cable force distribution, $\mathbf{f}_{c}$, using the closed-form method of Equation (8) whether the position of the end-effector is included in the wrench-feasible workspace. If the results of $f_{c}$ were included between the minimum and maximum feasible forces, $f_{\min }$ and $f_{\max }$, the end-effector's position used in Equation (8) was considered as a wrench-feasible workspace. $\mathbf{A}^{\mathrm{T}}$ is calculated by using the position of the end-effector, $\mathbf{p}$ (Equations (1)-(3)). Meanwhile, $\mathbf{A}^{+\mathrm{T}}$ is the pseudo-inverse of $\mathbf{A}^{\mathrm{T}}$, and $\mathbf{w}_{e}$ is the end-effector's external wrench vector, including its mass. The end-effector's position indicates the position of its bottom tip, location of the integrated printing nozzle; the boundary of the wrench-feasible workspace defined the possible ranges of the position of the end-effector along $x, y, z$ axes.

The initial wrench-feasible workspace was obtained by first placing $\mathbf{a}_{i}$, the $i$-th cable connection point on the external frame, at each corner of the top plane as the initial values (Table 1). Next, the locations of $\mathbf{a}_{i}$ were examined to determine how the locations of $\mathbf{a}_{i}$ can change the volume of the wrench-feasible workspace. The minimum and maximum cable forces, $f_{\min }$ and $f_{\max }$, for calculating the cable force distribution in Equation (8) were determined as $10 \mathrm{~N}$ and $200 \mathrm{~N}$, respectively, considering the dimensions of the potential 3D construction printing testbed's external frame was $2 \times 2 \times 2 \mathrm{~m}^{3}$ $\left(8 \mathrm{~m}^{3}\right)$. The mass of the retractable beam-type end-effector is assumed to be $10 \mathrm{~kg}$, which includes the retractable mechanism and 3D printing module. By using the initial locations of $\mathbf{a}_{i}$ and the maximum and minimum cable forces, the initial workspace volume was $2.96 \mathrm{~m}^{3}$, and it occupied approximately $37 \%$ of the external frame's volume (Figure 6).

Table 1. Initial dimensions (in meter) of a CDPR with a retractable beam-type end-effector.

\begin{tabular}{cccccccc}
\hline $\mathbf{a}_{\boldsymbol{i}}$ & $\boldsymbol{x}$ & $\boldsymbol{y}$ & $\boldsymbol{z}$ & $\mathbf{b}_{\boldsymbol{i}}$ & $\boldsymbol{x}$ & $\boldsymbol{y}$ & $\boldsymbol{z}$ \\
\hline $\mathbf{a}_{1}$ & 1.0 & 1.0 & 2.0 & $\mathbf{b}_{1}$ & 0.02 & 0.02 & 1.8 \\
$\mathbf{a}_{2}$ & 1.0 & -1.0 & 2.0 & $\mathbf{b}_{2}$ & 0.02 & -0.02 & 1.8 \\
$\mathbf{a}_{3}$ & -1.0 & -1.0 & 2.0 & $\mathbf{b}_{3}$ & -0.02 & -0.02 & 1.8 \\
$\mathbf{a}_{4}$ & -1.0 & 1.0 & 2.0 & $\mathbf{b}_{4}$ & -0.02 & 0.02 & 1.8 \\
$\mathbf{a}_{5}$ & 1.0 & 1.0 & 2.0 & $\mathbf{b}_{5}$ & 0.02 & 0.02 & -0.2 \\
$\mathbf{a}_{6}$ & 1.0 & -1.0 & 2.0 & $\mathbf{b}_{6}$ & 0.02 & -0.02 & -0.2 \\
$\mathbf{a}_{7}$ & -1.0 & -1.0 & 2.0 & $\mathbf{b}_{7}$ & -0.02 & -0.02 & -0.2 \\
$\mathbf{a}_{8}$ & -1.0 & 1.0 & 2.0 & $\mathbf{b}_{8}$ & -0.02 & 0.02 & -0.2 \\
\hline
\end{tabular}




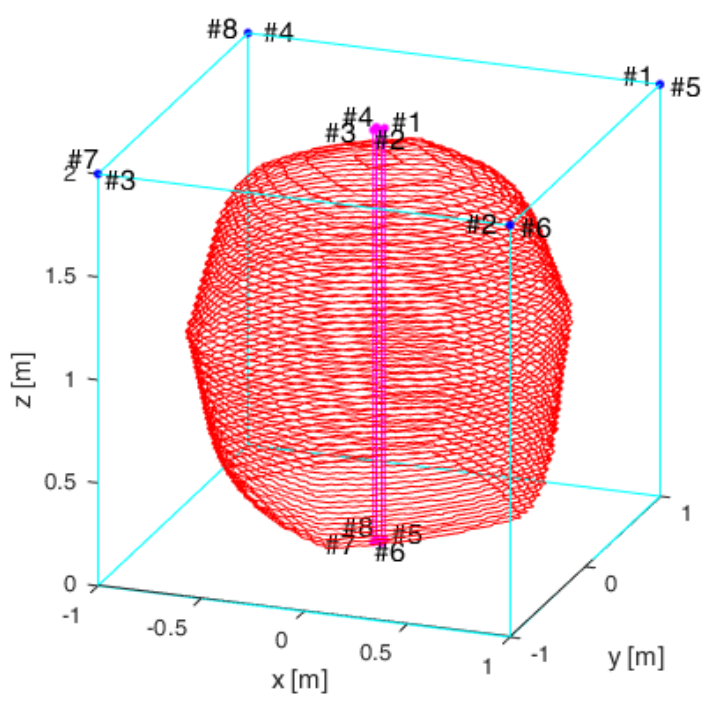

(a)

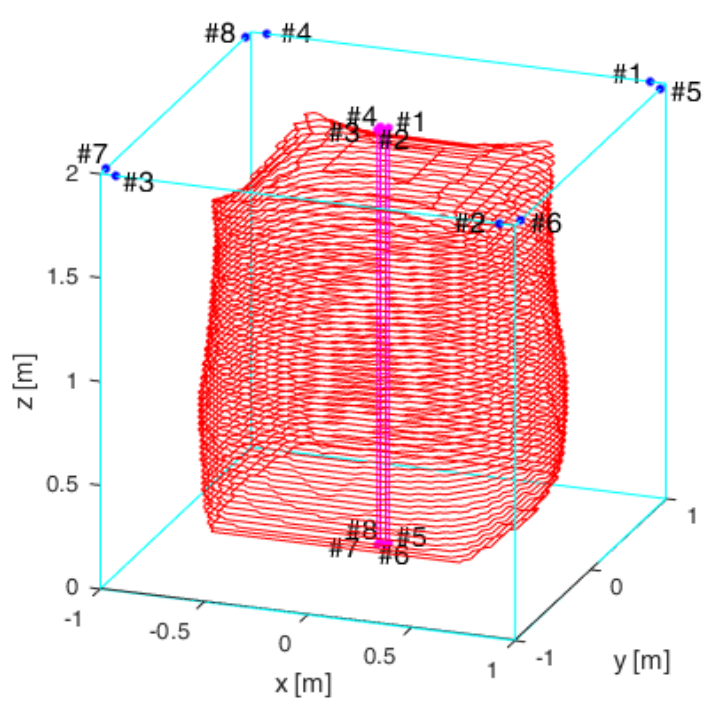

(b)

Figure 6. Wrench-feasible workspace (red lines) with the $\mathbf{a}_{i}$ (blue dots), $\mathbf{b}_{i}$ (magenta dots), and the external frame (cyan lines) on (a) initial wrench-feasible workspace with the initial locations of $\mathbf{a}_{i}$ and $\mathbf{b}_{i}$ and (b) improved wrench-feasible workspace when distances between the closest $\mathbf{a}_{i}$ are $0.0283 \mathrm{~m}$.

Changes in volume were observed at the locations of $\mathbf{a}_{i}$ varied along the horizontal edges of the external frame's top plane (Table 2). The variations of $\mathbf{a}_{i}$ are limited along the edges of the top plane to avoid cable interference with an object by maintaining cable connections (Figure 4). The workspace's volume decreased as soon as the distance between the neighboring $\mathbf{a}_{i}$ lengthened (Figure 6). When determining the locations of $\mathbf{a}_{i}$, considering the volume decrease in Figure 7, the minimum distance around $0.1 \mathrm{~m}$ was used to prevent the two $0.04 \mathrm{~m}$-diameter guide pulleys from colliding or overlapping during installation. Thus, the locations of $\mathbf{a}_{i}$ were $0.1 \mathrm{~m}$, and the corresponding $x, y$, and $z$ coordinates are shown in Table 3.

Table 2. The locations of $\mathbf{a}_{\mathrm{i}}$ when the distances are $0.0283(x=0.98)$ and $0.212(x=0.85)$, respectively.

\begin{tabular}{cccccccc}
\hline $\mathbf{a}_{\boldsymbol{i}}$ & $\boldsymbol{x}$ & $\boldsymbol{y}$ & $\boldsymbol{z}$ & $\mathbf{a}_{\boldsymbol{i}}$ & $\boldsymbol{x}$ & $\boldsymbol{y}$ & $\boldsymbol{z}$ \\
\hline $\mathbf{a}_{1}$ & 0.98 & 1.0 & 2.0 & $\mathbf{a}_{1}$ & 0.85 & 1.0 & 2.0 \\
$\mathbf{a}_{2}$ & 0.98 & -1.0 & 2.0 & $\mathbf{a}_{2}$ & 0.85 & -1.0 & 2.0 \\
$\mathbf{a}_{3}$ & -0.98 & -1.0 & 2.0 & $\mathbf{a}_{3}$ & -0.85 & -1.0 & 2.0 \\
$\mathbf{a}_{4}$ & -0.98 & 1.0 & 2.0 & $\mathbf{a}_{4}$ & -0.85 & 1.0 & 2.0 \\
$\mathbf{a}_{5}$ & 1.0 & 0.98 & 2.0 & $\mathbf{a}_{5}$ & 1.0 & 0.85 & 2.0 \\
$\mathbf{a}_{6}$ & 1.0 & -0.98 & 2.0 & $\mathbf{a}_{6}$ & 1.0 & -0.85 & 2.0 \\
$\mathbf{a}_{7}$ & -1.0 & -0.98 & 2.0 & $\mathbf{a}_{7}$ & -1.0 & -0.85 & 2.0 \\
$\mathbf{a}_{8}$ & -1.0 & 0.98 & 2.0 & $\mathbf{a}_{8}$ & -1.0 & 0.85 & 2.0 \\
\hline
\end{tabular}

Table 3. $\mathbf{a}_{i}$ without the collision of the guide pulleys. The distances between the neighboring $\mathbf{a}_{i}$ are $0.10 \mathrm{~m}$.

\begin{tabular}{cccc}
\hline $\mathbf{a}_{\boldsymbol{i}}$ & $\boldsymbol{x}$ & $\boldsymbol{y}$ & $\boldsymbol{z}$ \\
\hline $\mathbf{a}_{1}$ & 0.925 & 1.0 & 2.0 \\
$\mathbf{a}_{2}$ & 0.925 & -1.0 & 2.0 \\
$\mathbf{a}_{3}$ & -0.925 & -1.0 & 2.0 \\
$\mathbf{a}_{4}$ & -0.925 & 1.0 & 2.0 \\
$\mathbf{a}_{5}$ & 1.0 & 0.925 & 2.0 \\
$\mathbf{a}_{6}$ & 1.0 & -0.925 & 2.0 \\
$\mathbf{a}_{7}$ & -1.0 & -0.925 & 2.0 \\
$\mathbf{a}_{8}$ & -1.0 & 0.925 & 2.0 \\
\hline
\end{tabular}




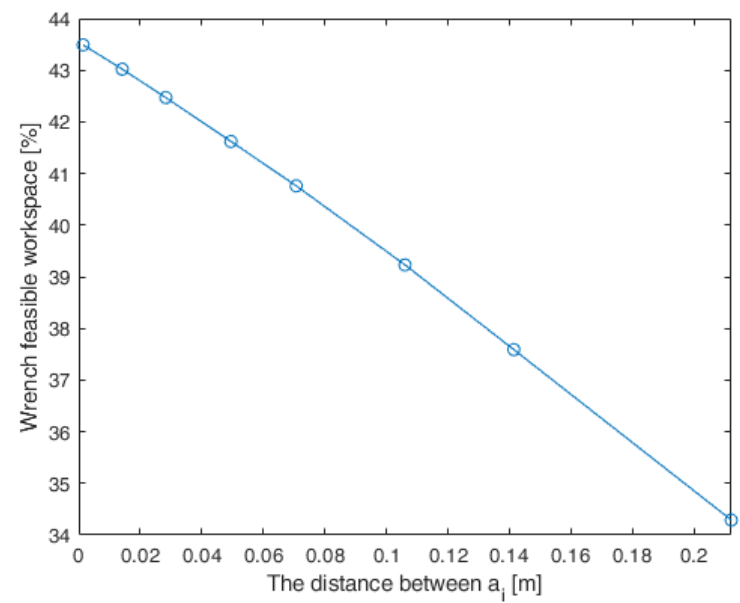

Figure 7. Volume percentages of wrench-feasible workspace compared to the volume of the external frame when the distance between ai increases.

The length of the retractable beam-type end-effector was modified using the previously determined $\mathbf{a}_{i}$ to investigate how the end-effector's change in length affected the volume of wrench-feasible workspace. In Figure 8, a decrease in the end-effector's length reduced the workspace's height and increased its width and depth because the angles of the cables along the horizontal plane became smaller while the horizontal component of the cable force grew larger.

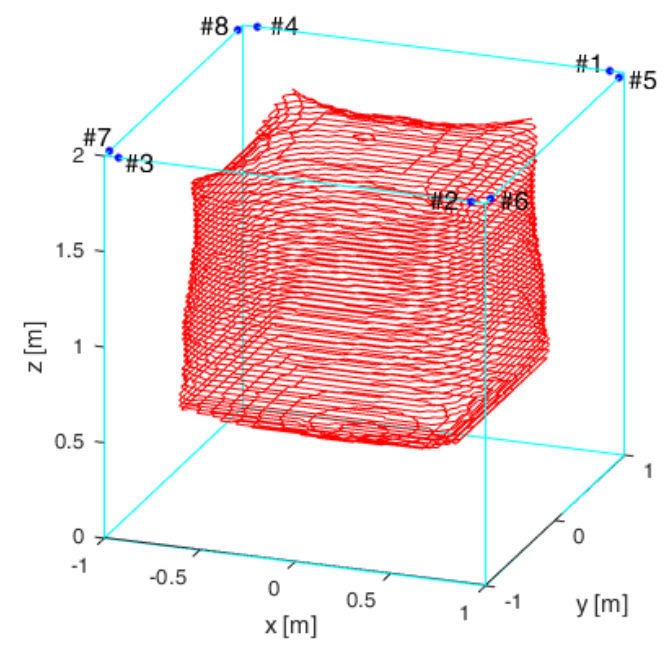

(a)

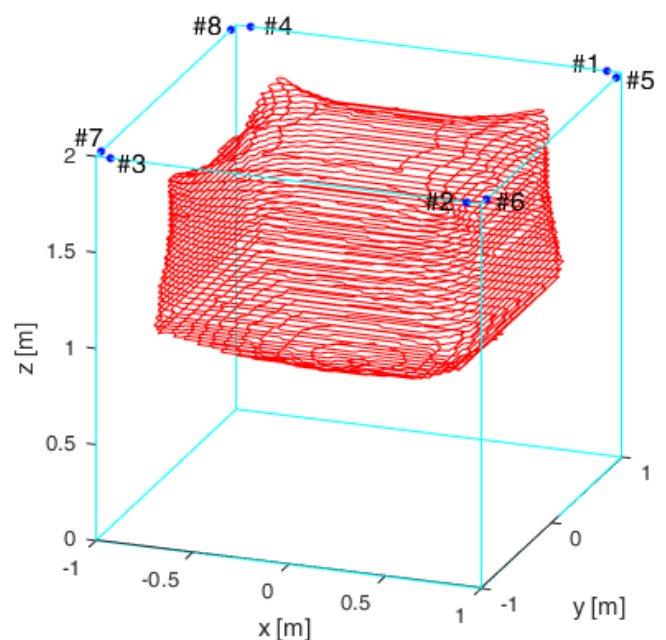

(b)

Figure 8. The volume changes of wrench-feasible workspace with retractable end-effector lengths at (a) $1.6 \mathrm{~m}$ and (b) $1.2 \mathrm{~m}$.

The volume changes of the workspace with the retracted lengths assume that when maximizing the volume of wrench-feasible workspace, the long end-effector is useful at low-level positions. In contrast, the short end-effector is effective at higher positions. The volume change with the retracted length along the z-axis is represented in Figure 9. Based on the volume change along a specific range of the z-axis, the retracted length can be continuously varied to maximize the overall workspace as the position of the end-effector changes along the z-axis. Thus, the end-effector's retracted length linear function was derived by utilizing an objective function to maximize the volume of the wrench-feasible workspace (Equations (13) and (14)). Figure 10 represents the change of the volume $V\left(z, l_{e}\right)$ according to the changes in the $\mathrm{z}$-axis and the retracted length. The linear equation of the retracted length $l_{e}(z)$ as 
a function of the z-axis position is derived as Equation (15) by the linear approximation of the points of maximum volume in Figure 10.

$$
\text { Maximize } V\left(z, l_{e}\right)=\int S_{f_{c}}\left(z, l_{e}\right) d z
$$

The wrench - feasible area of $x y$ plane $S_{f_{c}}\left(z, l_{e}\right)$ subject to $f_{\min }<\mathbf{f}_{c}\left(\mathbf{p}, l_{e}\right)<f_{\max }$ where $\mathbf{p}=[x, y, z]^{\mathrm{T}}$,

$$
l_{e}(z)=c_{r} z+d_{r}
$$

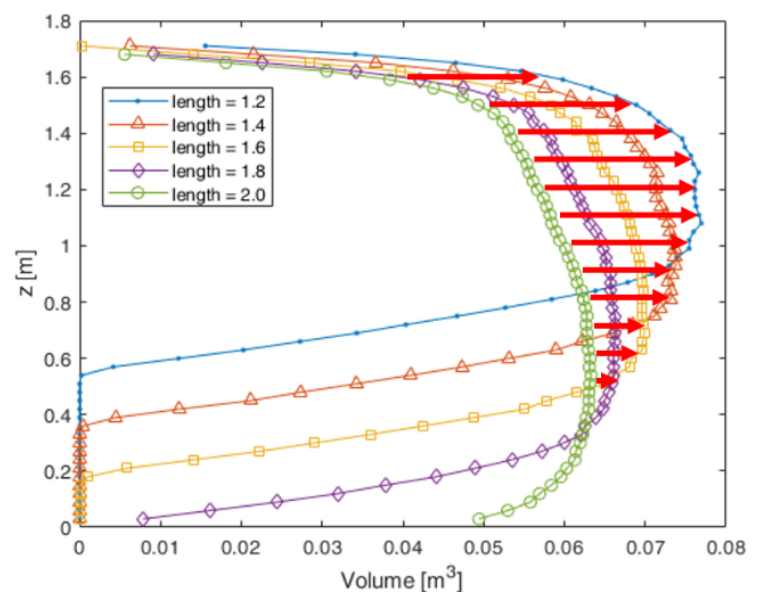

Figure 9. The volume changes of wrench-feasible workspace when decreasing the length of a retractable end-effector from 1.2 to $2.0 \mathrm{~m}$. The red arrows indicate the increases in volume that can be achieved by changing the length of the end-effector.

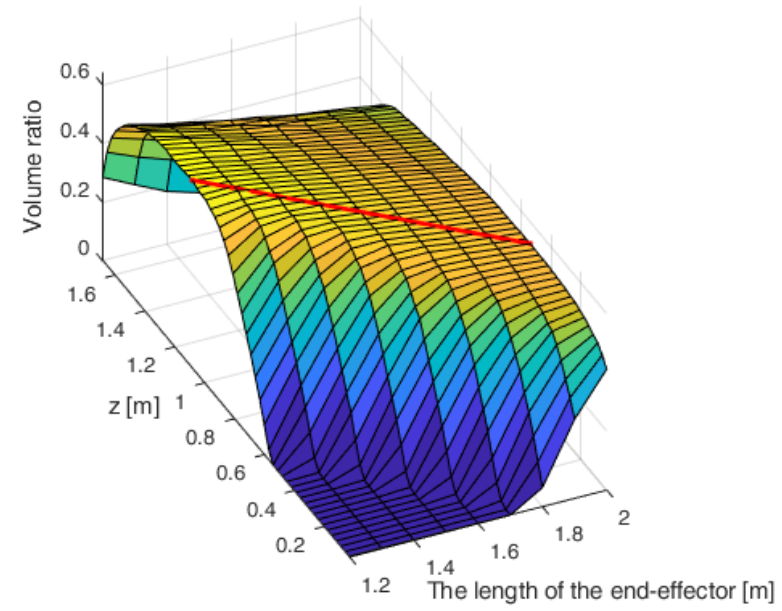

Figure 10. The changes in volume $V\left(z, l_{e}\right)$ according to the position changes in the z-axis and the retracted length of the end-effector with the linear function $l_{e}(z)$ (red line). Volume ratio is the ratio of the wrench-feasible volume $V\left(z, l_{l}\right)$ to the ideal volume of $2.0 \times 2.0 \times 0.03 \mathrm{~m}^{3}\left(0.12 \mathrm{~m}^{3}\right)$. Light yellow indicates the highest volume ratio of 0.64 , while dark blue represents the lowest value.

The values of $c_{r}$ and $d_{r}$ in Equation (15) were approximated as -0.675 and 1.929 , respectively. The parameters in Equation (16), $c_{r}, d_{r}$, and $l_{e}(z)$ can be used for representing the schematics of the retractable end-effector as shown in Figure 11. $c_{r}$ has a negative sign indicating the declined ratio of the change in the length of the end-effector to the change in the z-position. Thus, $c_{r}$ can be represented in Figure 11 as being multiplied by the z-position, such as $c_{r} z$. In addition, $d_{r}$ and $l_{e}(z)$ are represented as the maximum length of the end-effector and the retracted length of the end-effector respectively. 


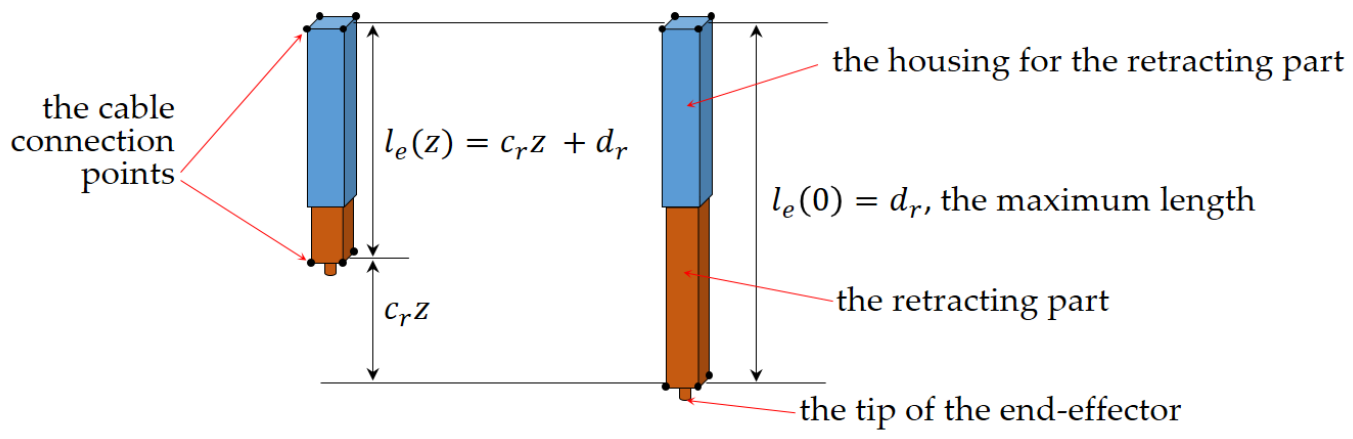

Figure 11. The schematics of the retractable end-effector: the left shows when the end-effector is retracted to $l_{e}(z)$ at a given z-position and the right represents the maximum length of the end-effector.

\section{Stiffness Analysis}

The stiffness analysis of wrench-feasible workspace was conducted by investigating the CDPR's natural frequencies to determine which motion is most vulnerable to external excitations. When 3D printing objects, if a CDPR has a considerably low natural frequency in the direction of motion, it would be easily affected by external excitations and print objects with uneven surfaces. The lowest natural frequencies were investigated along the boundary of the wrench-feasible workspace because the side walls or vertical columns of an object will be situated close to the boundary of the workspace while printing a large object. To calculate the natural frequencies of a CDPR, Equation (4) was used to solve an eigenvalue problem with zero-external forces (Equation (16)). The elements of the stiffness matrix $\mathbf{K}$ are the stiffness of each cable that varies as the cable length and the position of the end-effector change. The stiffness of each cable is calculated using Equation (17) with $\left|\mathbf{1}_{i}\right|$ as $i$-th cable length and $k_{s}$ as the material-specific stiffness of a polymer cable whose cable stiffness per unit length is $130,000 \mathrm{~N} / \mathrm{m}$.

$$
\begin{gathered}
\left(\mathbf{A}^{\mathrm{T}} \mathbf{K A}+\lambda^{2} \mathbf{M}_{e}\right) \mathbf{x}_{p}=0, \\
k_{i}=k_{s} /\left|\mathbf{l}_{i}\right|, \quad i=1, \ldots, 8
\end{gathered}
$$

Figure 12a shows the lowest natural frequencies of the retracted lengths, and Figure $12 \mathrm{~b}$ represents where the DOFs belong. In Figure 12b, the numbers 1,2, and 3 represent the translational DOFs of the $x$, $y$, and $z$ motions, while 4,5 , and 6 represent the rotational DOFs along the $x, y$, and $z$ axes, respectively. The lowest natural frequencies are around $1.2 \mathrm{~Hz}$, which mostly belong to the translational motion in the z-direction, and the rest occur in three rotational DOFs (Figure 12). The geometric parameters of the end-effector were adjusted to have a more vertically inclined cable, which contributed to the CDPR's increase in stiffness to improve the low natural frequencies. Each parameter was adjusted asymmetrically by increasing the end-effector's top width (in the $x$-direction) and bottom depth (in the $y$-direction) because the identical changes in geometric shape also improved the natural frequencies while inducing a smaller workspace volume. As shown in the simulation results, an increase of $0.08 \mathrm{~m}$ and $0.16 \mathrm{~m}$ in Figures 13 and 14, respectively, demonstrate that most of the natural frequencies increased by more than $8 \mathrm{~Hz}$. However, the asymmetrical changes also induced a $3 \%$ decrease in workspace volume. Thus, improving the natural frequency by adjusting the geometric parameters of the end-effector will be studied further in the future based on the experimental results of the frequencies of disturbance. 


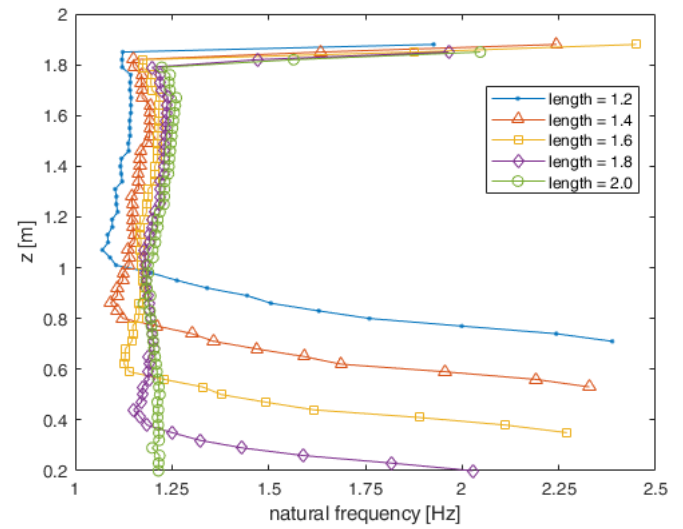

(a)

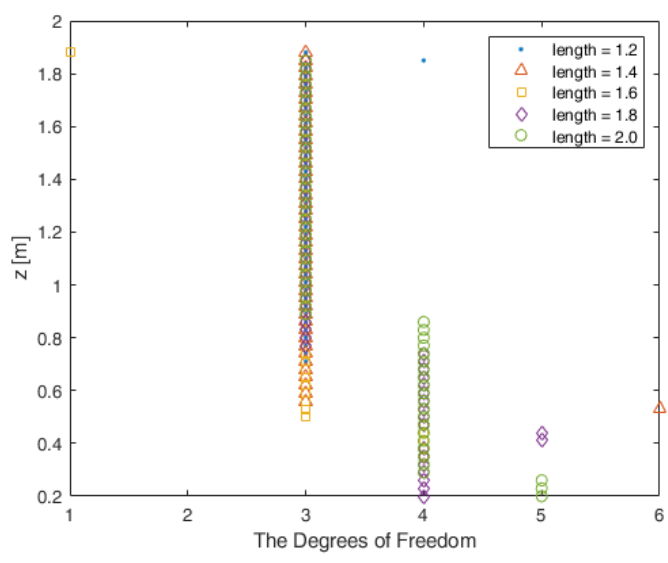

(b)

Figure 12. The (a) lowest natural frequencies (b) their degrees of freedom along the boundary of wrench-feasible workspace with the different lengths of the retractable end-effector from 1.2 to $2.0 \mathrm{~m}$. Most of the lowest natural frequencies belong to the translational $\mathrm{z}$ motion.

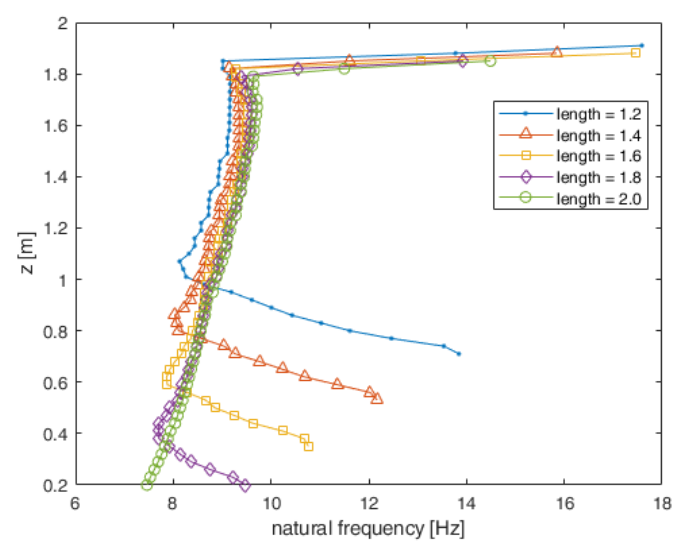

(a)

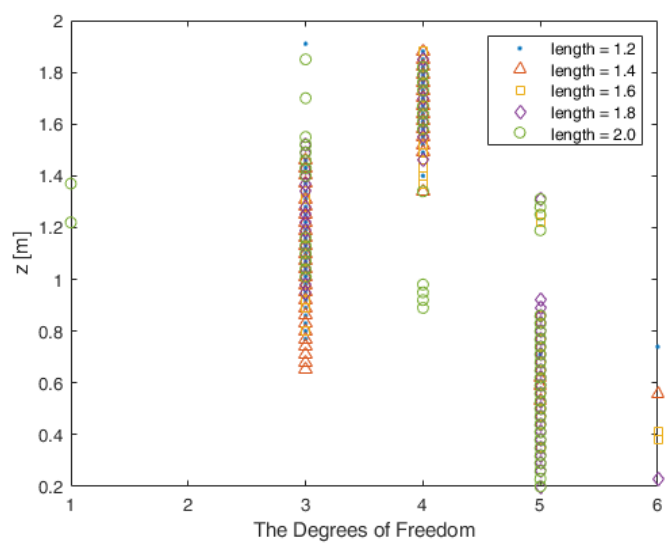

(b)

Figure 13. The increases in the (a) lowest natural frequencies and (b) their degrees of freedom along the boundary of wrench-feasible workspace from 7 to $17 \mathrm{~Hz}$. The lowest natural frequencies belong to the translated $\mathrm{z}$ motion and the rotational motions.

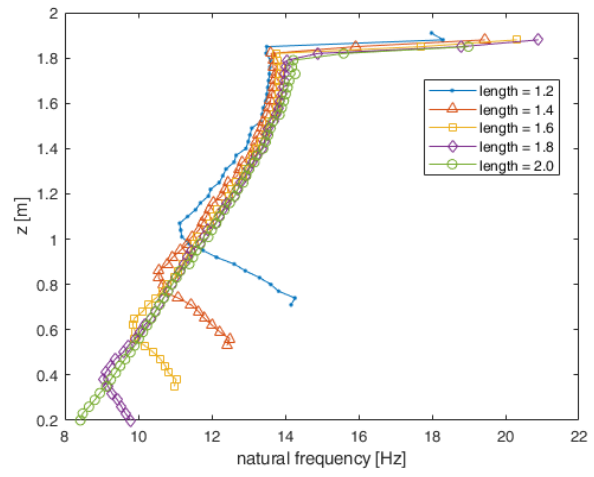

(a)

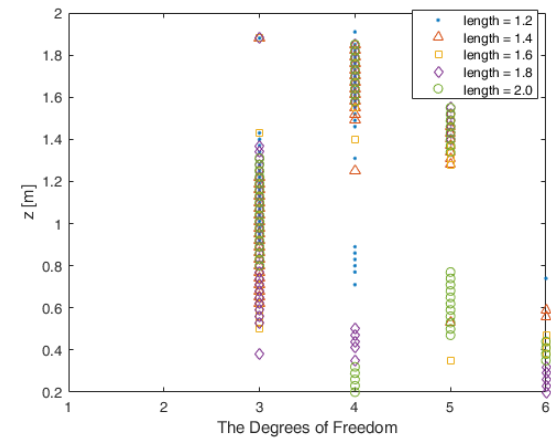

(b)

Figure 14. The increases in the (a) lowest natural frequencies and (b) their degrees of freedom along the boundary of wrench-feasible workspace from 8 to $21 \mathrm{~Hz}$. The lowest natural frequencies belong to the translated $\mathrm{z}$ motion and the rotational motions. 


\section{Conclusions and Future Works}

In this study, wrench-feasible workspace and stiffness analyses were conducted to investigate the effectiveness of a retractable beam-type end-effector for 3D construction printing. Cable connection types and the locations of cable connection points were determined to minimize cable interference with the printed objects within the workspace and maximize workspace volume, respectively. The wrench-feasible workspace can be expanded by varying the length of the retractable end-effector over the workspace, while the stiffness analysis shows that the lowest natural frequencies can be improved by adjusting the geometric parameters of the end-effector while considering disturbance frequencies.

In the future, the retractable beam-type end-effector for a 3D printing CDPR will be implemented. The CDPR testbed with a retractable end-effector's performance will also be investigated in terms of cable interference and the size and quality of various 3D printed objects.

Funding: This research was funded by the Basic Science Research Program through the National Research Foundation of Korea (NRF) and by the Ministry of Education, grant number NRF-2018R1C1B5086505.

Conflicts of Interest: The author declares no conflict of interest.

\section{References}

1. Duda, T.; Raghavan, L. 3D metal printing technology. IFAC-PapersOnline 2016, 49, 103-110. [CrossRef]

2. Buchanan, C.; Gardner, L. Metal 3D printing in construction: A review of methods, research, applications, opportunities and challenges. Eng. Struct. 2019, 180, 332-348. [CrossRef]

3. Gupta, S.; Bissoyi, A.; Bit, A. A review on 3D printable techniques for tissue engineering. Bionanoscience 2018, 8, 868-883. [CrossRef]

4. Valente, M.; Sibai, A.; Sambucci, M. Extrusion-based additive manufacturing of concrete products: Revolutionizing and remodeling the construction industry. J. Compos. Sci. 2019, 3, 88. [CrossRef]

5. Mechtcherine, V.; Nerella, V.; Will, F.; Näther, M.; Otto, J.; Krause, M. Large-scale digital concrete construction-CONPrint3D concept for on-site, monolithic 3D-printing. Autom. Constr. 2019, 107, 102933. [CrossRef]

6. Izard, J.; Dubor, A.; Hervé, P.; Cabay, E.; Cull, D.; Rodriguez, M.; Barrado, M. Large-scale 3D printing with cable-driven parallel robots. Constr. Robot. 2017, 1, 69-76. [CrossRef]

7. Zhong, Y.; Qian, S. A Cable-Driven Parallel Robot for 3D Printing. In Proceedings of the 2018 IEEE International Conference on Mechatronics, Robotics and Automation (ICMRA), Hefei, China, 18-21 May 2018; IEEE: Piscataway, NJ, USA; pp. 199-203.

8. Qian, S.; Bao, K.; Wang, N.; Zi, B. Kinematic calibration of a cable-driven parallel robot for 3D printing. Sensors 2018, 18, 2898. [CrossRef] [PubMed]

9. Bosscher, P.; Williams, R.; Bryson, L.; Castro-Lacouture, D. Cable-suspended robotic contour crafting system. Autom. Constr. 2007, 17, 45-55. [CrossRef]

10. Barnett, E.; Gosselin, C. Large-scale 3D printing with a cable-suspended robot. Addit. Manuf. 2015, 7, $27-44$. [CrossRef]

11. Zhang, Y.; Zhang, Y.; Dai, X.; Yang, Y. Workspace Analysis of a Novel 6-DOF Cable-Driven Parallel Robot. In Proceedings of the 2009 IEEE International Conference on Robotics and Biomimetics (ROBIO), Guilin, China, 19-23 December 2009; IEEE: Pistacaway, NJ, USA, 2009; pp. 2403-2408.

12. Gagliardini, L.; Gouttefarde, M.; Caro, S. Determination of a dynamic feasible workspace for cable-driven parallel robots. In Advances in Robot Kinematics; Lennarčič, J., Siciliano, B., Eds.; Springer International Publishing: New York, NY, USA, 2016; Volume 4, pp. 361-370.

13. Heo, J.-M.; Park, B.-J.; Park, J.-O.; Kim, C.-S.; Jung, J.; Park, K.-S. Workspace and stability analysis of a 6-DOF cable-driven parallel robot using frequency-based variable constraints. J. Mech. Sci. Technol. 2018, 32, 1345-1356. [CrossRef]

14. Boumann, R.; Bruckmann, T. Real-time cable force calculation beyond the wrench-feasible workspace. Robotics 2020, 9, 41. [CrossRef]

15. Yuan, H.; Courteille, E.; Deblaise, D. Static and dynamic stiffness analyses of cable-driven parallel robots with non-negligible cable mass and elasticity. Mech. Mach. Theory 2015, 85, 64-81. [CrossRef] 
16. Surdilovic, D.; Radojicic, J.; Krüger, J. Geometric stiffness analysis of wire robots: A mechanical approach. In Cable-Driven Parallel Robots. Mechanisms and Machine Science; Bruckmann, T., Pott, A., Eds.; Springer: Berlin/Heidelberg, Germany, 2013; Volume 12, pp. 389-404.

17. Kraus, W. Force Control of Cable-Driven Parallel Robots. Ph.D. Thesis, University of Stuttgart, Stuttgart, Germany, 2015.

18. Williams, R.L., II; Xin, M.; Bosscher, P. Contour-crafting-cartesian-cable robot system concepts: Workspace and stiffness comparisons. In Proceedings of the ASME International Design Technical Conferences, Brooklyn, NY, USA, 3-6 August 2018; ASME: New York, NY, USA, 2018; pp. 31-38.

19. Pott, A. Cable-Driven Parallel Robots: Theory and Application, 1st ed.; Springer International Publishing AG: Cham, Switzerland, 2018; pp. 89-91.

(C) 2020 by the author. Licensee MDPI, Basel, Switzerland. This article is an open access article distributed under the terms and conditions of the Creative Commons Attribution (CC BY) license (http://creativecommons.org/licenses/by/4.0/). 